\title{
Distinguishing partitions and asymmetric uniform hypergraphs*
}

\author{
M. N. Ellingham, Justin Z. Schroeder \\ Department of Mathematics, 1326 Stevenson Center \\ Vanderbilt University, Nashville, TN 37240, USA
}

Received 30 June 2010, accepted 30 October 2010, published online 23 March 2011

\begin{abstract}
A distinguishing partition for an action of a group $\Gamma$ on a set $X$ is a partition of $X$ that is preserved by no nontrivial element of $\Gamma$. As a special case, a distinguishing partition of a graph is a partition of the vertex set that is preserved by no nontrivial automorphism. In this paper we provide a link between distinguishing partitions of complete equipartite graphs and asymmetric uniform hypergraphs. Suppose that $m \geq 1$ and $n \geq 2$. We show that an asymmetric $n$-uniform hypergraph with $m$ edges exists if and only if $m \geq f(n)$, where $f(2)=f(14)=6, f(6)=5$, and $f(n)=\left\lfloor\log _{2}(n+1)\right\rfloor+2$ otherwise. It follows that a distinguishing partition of $K_{m(n)}=K_{n, n, \ldots, n}$, or equivalently for the wreath product action $S_{n} \operatorname{Wr} S_{m}$, exists if and only if $m \geq f(n)$.

Keywords: Complete equipartite graph, distinguishing number, distinguishing partition, asymmetric uniform hypergraph.
\end{abstract}

Math. Subj. Class.: 05C25, 05C65, 20B25

\section{Introduction}

A coloring of the vertices of a graph $G, c: V(G) \rightarrow\{1,2, \ldots, r\}$, is said to be $r$ distinguishing if there are no nontrivial color-preserving automorphisms of $G$. The distinguishing number $D(G)$ of $G$ is the smallest $r$ for which such a coloring exists. Let $V(G)=\left\{v_{1}, v_{2}, \ldots, v_{n}\right\}$; the coloring $c\left(v_{i}\right)=i$ is trivially $n$-distinguishing, so the distinguishing number is well-defined for all graphs. Albertson and Collins introduced these ideas in [1]; since then this topic has been well studied (c.f. [2, 4, 7, 9, 10, 12]).

These ideas were generalized to group actions by Tymoczko in [13]. Let $\Gamma$ be a group acting on a set $X$. A coloring of $X, c: X \rightarrow\{1,2, \ldots, r\}$, is $r$-distinguishing if the only

\footnotetext{
* In memory of Mike Albertson.

E-mail addresses: mark.ellingham@vanderbilt.edu (M. N. Ellingham), justin.z.schroeder@vanderbilt.edu (Justin Z. Schroeder)
} 


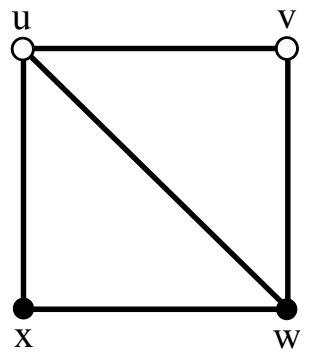

Figure 1: The automorphism $(u w)(v x)$ preserves the partition but not the coloring.

element in $\Gamma$ that preserves the coloring is the identity. For an action on a finite set $X$, a distinguishing coloring exists if and only if the action is faithful (only the identity fixes all elements of $X$ ). The distinguishing number of the action of $\Gamma$ on $X$, denoted $D_{\Gamma}(X)$, is the smallest $r$ for which such a coloring exists. Thus, $D(G)=D_{\text {Aut } G}(V(G))$. For further study in the setting of group actions see [5, 6, 11, 14].

Colorings of a set $X$ are closely related to partitions. Any coloring of $X$ gives a partition of $X$ into color classes, while any partition yields colorings by assigning a unique color to the elements of each part. If a group $\Gamma$ acts on $X$, each element of $\Gamma$ that preserves a coloring also preserves the associated partition, but the converse is not necessarily true. For example, the only graph automorphism that preserves the coloring in Figure 1 is the identity (so it is a distinguishing coloring), but the automorphism $(u w)(v x)$ preserves the color class partition $\{\{u, v\},\{w, x\}\}$.

Therefore, the idea of a distinguishing coloring may be modified to give the related but not identical idea of a distinguishing partition. A partition of $X$ is a distinguishing partition if the only element in $\Gamma$ that preserves the partition is the identity. To state this formally, suppose $\gamma \in \Gamma$. For any $S=\left\{x_{1}, x_{2}, \ldots, x_{s}\right\} \subseteq X$, let $\gamma^{\prime}(S)=\left\{\gamma\left(x_{1}\right)\right.$, $\left.\gamma\left(x_{2}\right), \ldots, \gamma\left(x_{s}\right)\right\}$. Moreover, for any collection $\mathcal{S}=\left\{S_{1}, S_{2}, \ldots, S_{t}\right\}$ of subsets of $X$, let $\gamma^{\prime \prime}(\mathcal{S})=\left\{\gamma^{\prime}\left(S_{1}\right), \gamma^{\prime}\left(S_{2}\right), \ldots, \gamma^{\prime}\left(S_{t}\right)\right\}$. Then a partition $\mathcal{S}$ of $X$ is distinguishing if $\gamma \in \Gamma$ and $\gamma^{\prime \prime}(\mathcal{S})=\mathcal{S}$ imply that $\gamma$ is the identity. In a graph, this translates to a partition of $V(G)$ such that there are no nontrivial automorphisms that preserve this partition.

Distinguishing partitions seem to be a natural concept, as other important ideas for group actions, such as primitivity, are defined in terms of the effect of elements of $\Gamma$ on partitions of $X$. Unlike distinguishing colorings, not all faithful group actions or graphs admit a distinguishing partition. For example, if $n \geq 2$ then $K_{n}$ does not. Thus, a fundamental question for a faithful group action or graph is whether or not it has a distinguishing partition.

This appears to be a difficult question. In this paper we explore some general properties of distinguishing partitions. Then we show that even for the complete equipartite graph $K_{m(n)}=K_{n, n, \ldots, n}$ (having $m$ parts of size $n$ ) it is not totally straightforward to say when it has a distinguishing partition. For a fixed $n$, we provide lower bounds for $m$ and show these are best possible. To arrive at these bounds, a connection to asymmetric uniform hypergraphs is established.

Despite their simple structure, complete equipartite graphs are an interesting family for our purposes because they have a high degree of symmetry that is difficult to destroy. They are ultrahomogeneous in the sense that every isomorphism between two induced subgraphs 
extends to an automorphism of the whole graph, making it very hard to distinguish one part of the graph from another. Gardiner [8] showed that, with two small exceptions, the only ultrahomogeneous graphs are the complete equipartite graphs and their complements.

\section{Hypergraphs and Two General Properties}

A hypergraph $H$ is a triple $(V(H), E(H), I(H))$, where $V(H)$ is a finite set of elements called vertices, $E(H)$ is a finite set of elements called edges, and $I(H) \subseteq V(H) \times E(H)$ is the incidence relation. If $(v, e) \in I(H)$, then we say the vertex $v$ is incident with the edge $e$. Often the incidence relation $I(H)$ is presented as an incidence matrix $M(H)=\left(m_{i j}\right)$, with rows indexed by vertices $v_{i}$ and columns indexed by edges $e_{j}$. The entry $m_{i j}=1$ if $\left(v_{i}, e_{j}\right) \in I(H)$, and $m_{i j}=0$ otherwise. If $v$ is incident with exactly $n$ edges, then we say the degree of $v$ is $n$; if all vertices $v \in V(H)$ have degree $n$, then $H$ is $n$-regular. Similarly, if there are exactly $n$ vertices incident with an edge $e$, then we say the size of $e$ is $n$; if all edges $e \in E(H)$ have size $n$, then $H$ is $n$-uniform. A graph is simply a 2-uniform hypergraph.

Define the sets $E_{H}(v)=\{e \in E(H) \mid(v, e) \in I(H)\}$ and $V_{H}(e)=\{v \in V(H) \mid$ $(v, e) \in I(H)\}$ (we simply write $E(v)$ and $V(e)$ if $H$ is understood). Two vertices $v_{1}, v_{2} \in$ $V(H)$ are said to be duplicate vertices if $E\left(v_{1}\right)=E\left(v_{2}\right)$; similarly two edges $e_{1}, e_{2} \in$ $E(H)$ are said to be duplicate edges if $V\left(e_{1}\right)=V\left(e_{2}\right)$. A hypergraph is vertex-simple or edge-simple if there are no duplicate vertices or duplicate edges, respectively.

While we define a hypergraph as an incidence structure, it is sometimes convenient to consider a hypergraph (particularly an edge-simple one) as a set structure where we identify the edge $e$ with the set $V(e)$. We will at various times consider a hypergraph as a set structure without explicitly making the transition.

An automorphism of a hypergraph $H$ is a pair $(\pi, \sigma)$, where $\pi$ is a permutation of $V(H)$ and $\sigma$ is a permutation of $E(H)$ such that $(v, e) \in I(H)$ if and only if $(\pi(v), \sigma(e)) \in I(H)$ for all $v \in V(H)$ and for all $e \in E(H)$. The automorphisms of $H$ form a group under composition, denoted Aut $H$. The image of the projection $(\pi, \sigma) \mapsto \pi$ is denoted Aut $_{V} H$, while the image of the projection $(\pi, \sigma) \mapsto \sigma$ is denoted $\operatorname{Aut}_{E} H$. The members of Aut $_{V} H$ and Aut $_{E} H$ are called vertex automorphisms and edge automorphisms, respectively. If Aut $H$ is the trivial group, we say $H$ is asymmetric. A pair of duplicate vertices or edges gives rise to an automorphism that simply swaps these vertices or edges, so an asymmetric hypergraph is necessarily vertex-simple and edge-simple.

The dual of $H$, denoted by $H^{*}$, is a hypergraph $\left(V\left(H^{*}\right), E\left(H^{*}\right), I\left(H^{*}\right)\right)$, where $V\left(H^{*}\right)=E(H), E\left(H^{*}\right)=V(H)$, and $I\left(H^{*}\right)=\{(e, v) \mid(v, e) \in I(H)\}$. In other words, the dual $H^{*}$ swaps the vertices and edges of $H$. The map $(\pi, \sigma) \in$ Aut $H \mapsto$ $(\sigma, \pi) \in$ Aut $H^{*}$ gives rise to the following observation.

Observation 2.1. For every hypergraph $H$, Aut $H \cong$ Aut $H^{*}$.

Many vertex properties of hypergraphs translate naturally to edge properties using the dual construction. For example, $H$ is $n$-regular if and only if $H^{*}$ is $n$-uniform, and $H$ is vertex-simple if and only if $H^{*}$ is edge-simple. In addition, we have the following lemma.

Lemma 2.2. Let $H$ be a hypergraph.

1. If $H$ is edge-simple, then the projection $(\pi, \sigma) \mapsto \pi$ is an isomorphism from Aut $H$ to $\mathrm{Aut}_{V} H$. 
2. If $H$ is vertex-simple, then the projection $(\pi, \sigma) \mapsto \sigma$ is an isomorphism from Aut $H$ to $\mathrm{Aut}_{E} H$.

Proof. (1) We know the given projection is a surjective homomorphism, so it remains to show that it is injective. Assume $\left(\pi, \sigma_{1}\right),\left(\pi, \sigma_{2}\right) \in$ Aut $H$ are two elements that project to $\pi$. Since $\left(\pi, \sigma_{1}\right)$ is an automorphism, we must have $V\left(\sigma_{1}(e)\right)=\pi(V(e))$ for all $e \in$ $E(H)$. Similarly we must have $V\left(\sigma_{2}(e)\right)=\pi(V(e))$ for all $e \in E(H)$. But this implies $V\left(\sigma_{1}(e)\right)=V\left(\sigma_{2}(e)\right)$ for all $e \in E(H)$. Since there are no duplicate edges, this means $\sigma_{1}(e)=\sigma_{2}(e)$ for all $e \in E(H)$. Hence $\sigma_{1}=\sigma_{2}$, and the projection is injective as desired.

(2) This follows by applying part (i) to $H^{*}$ and using Observation 2.1.

Suppose we have a function $f: X \rightarrow Y$. A subset $\left\{x_{1}, x_{2}, \ldots, x_{k}\right\} \subseteq X$ is mapped to the subset $\left\{f\left(x_{1}\right), f\left(x_{2}\right), \ldots, f\left(x_{k}\right)\right\} \subseteq Y$. This induces a function $f^{\prime}: 2^{X} \rightarrow 2^{Y}$ from subsets of $X$ to subsets of $Y$. Similarly $f^{\prime}$ induces a function $f^{\prime \prime}: 2^{2^{X}} \rightarrow 2^{2^{Y}}$ from collections of subsets of $X$ to collections of subsets of $Y$. Moreover, if $f$ is injective, then so are $f^{\prime}$ and $f^{\prime \prime}$. Commonly $f^{\prime}$ and $f^{\prime \prime}$ are just denoted by $f$, but for our purposes it will be helpful to distinguish between $f, f^{\prime}$ and $f^{\prime \prime}$.

The notation above, together with Lemma 2.2, provides a natural way to talk about automorphisms of a hypergraph as a set structure. If $H$ is an edge-simple hypergraph and $(\pi, \sigma) \in$ Aut $H$, then we must have $\sigma=\left.\pi^{\prime}\right|_{E(H)}$. Additionally, a permutation $\pi$ of $V(H)$ is an automorphism of $H$ if and only if $\pi^{\prime \prime}(E(H))=E(H)$. Equivalently, a permutation $\pi$ of $V(H)$ is an automorphism of $H$ if and only if $\pi^{\prime \prime}(E(v))=E(\pi(v))$ for all $v \in V(H)$. These facts will be useful in the arguments of Section 3.

Now we mention two general results, the first a positive result for both graphs and faithful group actions, and the second a negative result just for graphs.

Theorem 2.3. Suppose $|X| \geq 3$ and the action of $\Gamma$ on $X$ has distinguishing number at most 2. Then the action has a distinguishing partition.

Proof. If the distinguishing number is 1 then any partition is a distinguishing partition. Suppose the distinguishing number is 2 and there is a distinguishing coloring with color classes $C_{1}, C_{2}$ where $\left|C_{1}\right| \geq\left|C_{2}\right|$. Then $\left|C_{1}\right| \geq 2$. Thus, the partition consisting of $C_{1}$ together with $\left|C_{2}\right|$ singletons containing the elements of $C_{2}$ is a distinguishing partition, because any automorphism preserving this partition also preserves the distinguishing coloring.

This is useful, because there are a number of results showing that classes of graphs have distinguishing number 2. For example, Klavžar and Zhu and also Imrich and Klavžar $[10,12]$ give conditions on cartesian products and cartesian powers under which the distinguishing number is 2 ; all graphs satisfying their conditions then have a distinguishing partition.

Let $G$ be a graph (i.e., 2-uniform hypergraph). If $G$ is simple in the usual sense (i.e., edge-simple) then we will apply Lemma 2.2(i) and consider automorphisms as just permutations of $V(G)$. If $u v \in E(G)$, we say $u$ and $v$ are adjacent and write $u \sim v$. A subset of vertices $X \subseteq V(G)$ is a module if for every $v \in V(G) \backslash X$, either $x \sim v$ for all $x \in X$ or $x \not v$ for all $x \in X$. If in addition $X$ forms an independent set or clique, then $X$ is called an independent module or complete module, respectively. In general, large independent or complete modules prevent the existence of a distinguishing partition, as shown in the following result. 
Theorem 2.4. Let $G$ be a graph with $|V(G)|=n$. If $G$ contains an independent or complete module $X$ with $|X|>\frac{n+1}{2}$, then there exists no distinguishing partition of $G$.

Proof. Assume $G$ has a distinguishing partition. To avoid an automorphism that simply swaps two vertices in $X$, each vertex in $X$ must be in a different set of the partition, and at most one vertex in $X$ can be in a set by itself. Thus, every other vertex in $X$ must be in a partition with some element not in $X$, so $n \geq 2|X|-1$. But this implies $\frac{n+1}{2} \geq|X|$, a contradiction. Therefore, no distinguishing partition exists.

\section{Reduction to hypergraphs}

In this section we establish a connection between automorphisms of $K_{m(n)}$ preserving a partition of its vertices and automorphisms of certain hypergraphs.

Let $[k]$ denote the set $\{1,2, \ldots, k\}$. The graph $K_{m(n)}$ contains $m$ induced copies of $\overline{K_{n}}$, and we can let $V\left(K_{m(n)}\right)=[m] \times[n]$, where $(i, j)$ denotes the $j$ th vertex in the $i$ th copy of $\overline{K_{n}}$. Let $\rho: V\left(K_{m(n)}\right) \rightarrow[m]$ be the projection given by $\rho(i, j)=i$. In the graph $K_{m(n)}$, any automorphism permutes the $m$ copies of $\overline{K_{n}}$ along with independently permuting the vertices within each $\overline{K_{n}}$. Using the definitions of [3], we see that (after reversing coordinates in $V\left(K_{m(n)}\right)=[m] \times[n]$ ) the action of Aut $K_{m(n)}$ is just the wreath product action $S_{n} \mathrm{Wr} S_{m}$ on the set $[n] \times[m]$, where $S_{k}$ is the symmetric group acting on $[k]$.

The following theorem formalizes the link between asymmetric hypergraphs and distinguishing partitions of complete equipartite graphs. The idea is to project a partition of $V\left(K_{m(n)}\right)$ using $\rho^{\prime \prime}$ to obtain a hypergraph with vertex set $[m]$, or, conversely, to lift a hypergraph to obtain a partition. Intuitively it is not hard to see that the partition will be distinguishing if and only if the hypergraph is $n$-regular and asymmetric, but verifying this rigorously requires some technical arguments.

Theorem 3.1. The following are equivalent for $m, n \geq 1$ :

1. There exists a distinguishing partition of $K_{m(n)}$.

2. There exists a distinguishing partition of $m K_{n}$ (the union of $m$ disjoint copies of $\left.K_{n}\right)$.

3. There exists an asymmetric n-regular hypergraph with $m$ vertices.

4. There exists an asymmetric n-uniform hypergraph with $m$ edges.

5. There exists a distinguishing partition of the wreath product $S_{n} \mathrm{Wr} S_{m}$ acting on the set $[n] \times[m]$.

Proof. (1) $\Leftrightarrow(2)$ This follows because $m K_{n}=\overline{K_{m(n)}}$ and complementary graphs have the same automorphism group.

(1) $\Rightarrow$ (3) Assume $\mathcal{S}=\left\{S_{1}, S_{2}, \ldots, S_{r}\right\}$ is a distinguishing partition for $K_{m(n)}$. Thus, if $\alpha \in$ Aut $K_{m(n)}$ such that $\alpha^{\prime \prime}(\mathcal{S})=\mathcal{S}$, it follows that $\alpha=1_{[m] \times[n]}$.

For every $(i, j) \in V\left(K_{m(n)}\right)$, there exists a unique $k$ such that $(i, j) \in S_{k}$; define the function $g_{i}(j)=k$ for all $(i, j) \in V\left(K_{m(n)}\right)$. If two distinct vertices $\left(i, j_{1}\right)$ and $\left(i, j_{2}\right)$ both belong to $S_{k}$ then there is an automorphism of $K_{m(n)}$ swapping these two vertices and preserving $\mathcal{S}$, which is a contradiction. Therefore, each function $g_{i}$ is injective. We can define $g_{i}^{-1}(k)$ precisely when $i \in \rho^{\prime}\left(S_{k}\right)$, and

$$
(i, j) \in S_{k} \quad \Leftrightarrow \quad k=g_{i}(j) \quad \Leftrightarrow \quad j=g_{i}^{-1}(k) .
$$


Consider the set structure $H$ obtained by setting $V(H)=[m]$ and $E(H)=\rho^{\prime \prime}(\mathcal{S})$. Since each $g_{i}$ is injective, $n$ distinct elements of $\rho^{\prime \prime}(\mathcal{S})$ contain each $i \in[m]$, and so $H$ is an $n$-regular hypergraph.

We claim that $H$ is edge-simple, i.e., that no two distinct edges are equal as sets. Suppose $\rho^{\prime}\left(S_{k_{1}}\right)=\rho^{\prime}\left(S_{k_{2}}\right)$. Then for every $\left(i, j_{i, 1}\right) \in S_{k_{1}}$ there exists $\left(i, j_{i, 2}\right) \in S_{k_{2}}$. The map that swaps these two elements for all $i \in \rho^{\prime}\left(S_{k_{1}}\right)$ forms an automorphism $\alpha$ of $K_{m(n)}$ such that $\alpha^{\prime \prime}(\mathcal{S})=\mathcal{S}$. But this implies $\alpha=1_{[m] \times[n]}$; hence, $S_{k_{1}}=S_{k_{2}}$ and $k_{1}=k_{2}$. Thus, $H$ is edge-simple.

We want to show that Aut $H$ is trivial; since $H$ is edge-simple, it will suffice to show that Aut $_{V} H$ is trivial. Let $\pi \in \operatorname{Aut}_{V} H$. We know $\pi^{\prime \prime}(E(H))=\pi^{\prime \prime}\left(\rho^{\prime \prime}(\mathcal{S})\right)$, so we can define the permutation $p$ on $[r]$ by $\pi^{\prime}\left(\rho^{\prime}\left(S_{k}\right)\right)=\rho^{\prime}\left(S_{p(k)}\right)$. Set $\alpha(i, j)=$ $\left(\pi(i), g_{\pi(i)}^{-1}\left(p\left(g_{i}(j)\right)\right)\right)$. We claim that (1) $\alpha$ is well-defined, (2) $\rho \circ \alpha=\pi \circ \rho$, (3) $\alpha \in$ Aut $K_{m(n)}$, and $(4) \alpha^{\prime \prime}(\mathcal{S})=\mathcal{S}$.

For (1), it suffices to show that $g_{\pi(i)}^{-1}\left(p\left(g_{i}(j)\right)\right)$ is well-defined. This is true if and only if $\pi(i) \in \rho^{\prime}\left(S_{p\left(g_{i}(j)\right)}\right)=\pi^{\prime}\left(\rho^{\prime}\left(S_{g_{i}(j)}\right)\right)$, which is true if and only if $i \in \rho^{\prime}\left(S_{g_{i}(j)}\right)$. But $(i, j) \in S_{g_{i}(j)}$ by definition of $g_{i}$, so this is always true, and $\alpha$ is well-defined. To prove (2), note that $\rho \circ \alpha(i, j)=\rho\left(\pi(i), g_{\pi(i)}^{-1}\left(p\left(g_{i}(j)\right)\right)\right)=\pi(i)=\pi(\rho(i, j))=\pi \circ \rho(i, j)$ for all $(i, j) \in V\left(K_{m(n)}\right)$. We know $\pi$ is a permutation of $[m]$, so to verify (3) it suffices to show that $j \mapsto g_{\pi(i)}^{-1}\left(p\left(g_{i}(j)\right)\right)$ is a permutation of $[n]$ for each $i \in[m]$. We already know $g_{i}, p$, and $g_{\pi(i)}^{-1}$ are injective, so the composition of these maps is also injective. Since $j \mapsto g_{\pi(i)}^{-1}\left(p\left(g_{i}(j)\right)\right)$ is an injective map from $[n]$ to $[n]$, it must be a permutation. Finally, consider (4). Suppose $(i, j) \in S_{k}$. Then $\alpha(i, j)=\left(\pi(i), g_{\pi(i)}^{-1}\left(p\left(g_{i}(j)\right)\right)\right)=$ $\left(\pi(i), g_{\pi(i)}^{-1}(p(k))\right) \in S_{p(k)}$, using $(*)$ twice. Thus, $\alpha^{\prime}\left(S_{k}\right) \subseteq S_{p(k)}$ for all $k$. But $\bigcup_{k=1}^{r} \alpha^{\prime}\left(S_{k}\right)=V\left(K_{m(n)}\right)=\bigcup_{k=1}^{r} S_{p(k)}$, so we must have $\alpha^{\prime}\left(S_{k}\right)=S_{p(k)}$ for all $k$, and $\alpha^{\prime \prime}(\mathcal{S})=\mathcal{S}$, as required.

From (3) and (4) it follows that $\alpha=1_{[m] \times[n]}$, and from (2) we learn that $\pi \circ \rho=$ $\rho \circ \alpha=\rho \circ 1_{[m] \times[n]}=\rho$. Thus, $\pi(i)=\pi(\rho(i, j))=\rho(i, j)=i$ for all $i \in[m]$, and hence $\pi=1_{[m]}$. This shows Aut $V H$ is trivial, as desired.

(3) $\Rightarrow(1)$ Assume there is an asymmetric $n$-regular hypergraph $H$ with $V(H)=[m]$; we know it must be edge-simple. Let the edges be denoted $T_{1}, T_{2}, \ldots, T_{r}$, and for each $i$ define the distinct numbers $g_{i}(1), g_{i}(2), \ldots, g_{i}(n)$ such that $E(i)=\left\{T_{g_{i}(1)}, T_{g_{i}(2)}, \ldots, T_{g_{i}(n)}\right\}$. For each $(i, j) \in[m] \times[n]$ replace $i$ in $T_{g_{i}(j)}$ by $(i, j)$; each edge $T_{k}$ now becomes a set $S_{k} \subseteq[m] \times[n]=V\left(K_{m(n)}\right)$. Let $\mathcal{S}=\left\{S_{1}, S_{2}, \ldots, S_{r}\right\}$; this collection partitions $V\left(K_{m(n)}\right)$. We see that $\rho^{\prime}\left(S_{k}\right)=T_{k}$ for all $k \in[r]$. Let $\alpha \in$ Aut $K_{m(n)}$ be an automorphism such that $\alpha^{\prime \prime}(\mathcal{S})=\mathcal{S}$. We want to show $\alpha=1_{[m] \times[n]}$.

We can write $\alpha(i, j)=\left(\pi(i), h_{i}(j)\right)$, where $\pi$ is a permutation of $[m]$ and each $h_{i}$ is a permutation of $[n]$. Then $\pi(\rho(i, j))=\pi(i)=\rho(\alpha(i, j))$, so that $\pi \circ \rho=\rho \circ \alpha$. Define the permutation $p$ on $[r]$ by $\alpha^{\prime}\left(S_{k}\right)=S_{p(k)}$. For each $k$,

$$
\begin{aligned}
\pi^{\prime}\left(T_{k}\right) & =\pi^{\prime}\left(\rho^{\prime}\left(S_{k}\right)\right)=(\pi \circ \rho)^{\prime}\left(S_{k}\right)=(\rho \circ \alpha)^{\prime}\left(S_{k}\right) \\
& =\rho^{\prime}\left(\alpha^{\prime}\left(S_{k}\right)\right)=\rho^{\prime}\left(S_{p(k)}\right)=T_{p(k)} .
\end{aligned}
$$

But then $\pi^{\prime \prime}(E(H))=E(H)$, so $\pi \in$ Aut $_{V} H$. By asymmetry of $H$, this means $\pi=1_{[m]}$. Thus, $p=1_{[r]}$ as well. Now for every $(i, j) \in S_{k}$, we have $\alpha(i, j)=\left(\pi(i), h_{i}(j)\right)=$ $\left(i, h_{i}(j)\right) \in \alpha^{\prime}\left(S_{k}\right)=S_{p(k)}=S_{k}$. However, $(i, j),\left(i, h_{i}(j)\right) \in S_{k}$ implies $j=h_{i}(j)$. It 


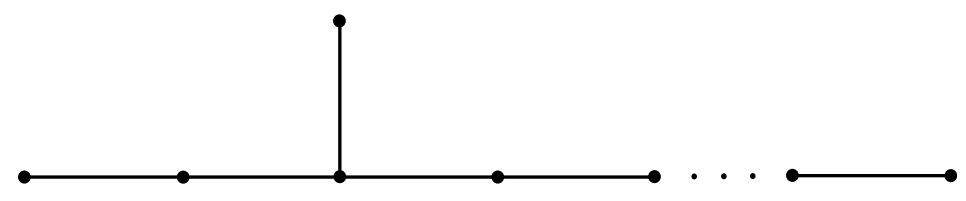

Figure 2: An asymmetric 2-uniform hypergraph with 6 or more edges.

follows that $h_{i}=1_{[n]}$ for all $i \in[m]$, so $\alpha=1_{[m] \times[n]}$. This shows $\mathcal{S}$ is a distinguishing partition for $K_{m(n)}$ as desired.

(3) $\Leftrightarrow(4)$ This follows from considering the dual hypergraph and Observation 2.1.

$(1) \Leftrightarrow(5)$ As discussed above, this action is precisely the automorphism group of $K_{m(n)}$, from which the result follows.

Henceforth we consider our problem as in Theorem 3.1(iv), in terms of asymmetric uniform hypergraphs. Note the restriction $m \geq 1$ in the theorem; this excludes trivial asymmetric uniform hypergraphs with no edges and one or no vertices. From the uniform hypergraph setting we are able to get a lower bound for $m$.

Corollary 3.2. Suppose that $m \geq 1$ and $n \geq 2$. If $H$ is an asymmetric $n$-uniform hypergraph with $m$ edges, then $m \geq\left\lceil\log _{2} n\right\rceil+1$.

Proof. Pick any edge $f$. Since $H$ is asymmetric, it must be vertex-simple, so the sets $E(u)$ must be distinct for all $u \in V(f)$. For every edge $g \in E(H) \backslash\{f\}$, either $g \in E(u)$ or $g \notin$ $E(u)$. Thus, there are at most $2^{m-1}$ possibilities for $E(u)$. Since they must all be distinct, it follows that $n=|V(f)| \leq 2^{m-1}$. Solving this inequality yields $m \geq\left\lceil\log _{2} n\right\rceil+1$.

The following small cases will be needed later in the paper.

\section{Theorem 3.3.}

1. There are no asymmetric 0- or 1-uniform hypergraphs with 2 or more edges.

2. There exists an asymmetric 2-uniform hypergraph with $m \geq 1$ edges if and only if $m \geq 6$.

Proof. (1) This is clear.

(2) An asymmetric 2-uniform hypergraph is simply a graph in the normal sense with a trivial automorphism group. It is not hard to show that every graph with fewer than 6 edges has some nontrivial automorphism. Furthermore, the tree obtained from joining copies of the linear graphs $P_{2}, P_{3}$, and $P_{m-2}, m \geq 6$, at a common endpoint yields an $m$-edge graph with trivial automorphism group (see Figure 2).

\section{The vertex and edge power complement}

To define the vertex power complement $H^{V P}$ of an edge-simple hypergraph $H$, we want to consider $H$ as a set structure, so assume $e=V(e)$ for all $e \in E(H)$. Let $V\left(H^{V P}\right)=$ $V(H)$, and let $E\left(H^{V P}\right)=2^{V(H)} \backslash E(H)$, where $2^{V(H)}$ is the power set of $V(H)$. This yields an edge-simple hypergraph $H^{V P}$ with the same vertex set as $H$ and $\left|E\left(H^{V P}\right)\right|=$ 
$2^{|V(H)|}-|E(H)|$. Moreover, if $H$ is $n$-regular, then $H^{V P}$ is $\left(2^{|V(H)|-1}-n\right)$-regular. Since $H$ and $H^{V P}$ are edge-simple, we have for any permutation $\pi$ of $V(H)$

$$
\begin{aligned}
\pi \in \mathrm{Aut}_{V} H & \Leftrightarrow \pi^{\prime \prime}(E(H))=E(H) \\
& \Leftrightarrow \pi^{\prime \prime}\left(E\left(H^{V P}\right)\right)=\pi^{\prime \prime}\left(2^{V(H)} \backslash E(H)\right)=2^{V(H)} \backslash E(H)=E\left(H^{V P}\right) \\
& \Leftrightarrow \pi \in \operatorname{Aut}_{V} H^{V P},
\end{aligned}
$$

which, together with Lemma 2.2(1), leads directly to the following observation.

Observation 4.1. For every edge-simple hypergraph $H$, Aut $H \cong \operatorname{Aut}_{V} H=\operatorname{Aut}_{V} H^{V P}$ $\cong$ Aut $H^{V P}$.

We can now define the edge power complement $H^{E P}$ of a vertex-simple hypergraph $H$ by $H^{E P}=\left(\left(H^{*}\right)^{V P}\right)^{*}$. In other words, we swap the vertices and edges, find the vertex power complement, then reverse the vertices and edges back to their original roles. If we think of our hypergraph in a slightly unusual way, identifying vertices with sets of edges, then $V\left(H^{E P}\right)=2^{E(H)} \backslash V(H)$ and $E\left(H^{E P}\right)=E(H)$. Moreover, if $H$ is $n$-uniform, then $H^{E P}$ is $\left(2^{|E(H)|-1}-n\right)$-uniform. As with the vertex power complement, the edge power complement preserves the automorphism group.

Corollary 4.2. For every vertex-simple hypergraph $H$, Aut $H \cong \operatorname{Aut}_{E} H=\operatorname{Aut}_{E} H^{E P}$ $\cong$ Aut $H^{E P}$.

Proof. This follows from Lemma 2.2(2), Observation 2.1, and Observation 4.1.

Example 4.3. $H^{V P}$ and $H^{E P}$ can be represented simply in terms of incidence matrices. Let $H$ be the hypergraph with incidence matrix $M(H)$ below.

$$
M(H)=\left(\begin{array}{lll}
1 & 1 & 0 \\
1 & 0 & 0 \\
0 & 1 & 1
\end{array}\right) .
$$

The hypergraph $H^{V P}$ is then given by the incidence matrix $M\left(H^{V P}\right)$ that consists of all binary columns not in $M(H)$. Likewise, the hypergraph $H^{E P}$ is given by the incidence matrix $M\left(H^{E P}\right)$ that consists of all binary rows not in $M(H)$.

$$
M\left(H^{V P}\right)=\left(\begin{array}{ccccc}
0 & 1 & 0 & 0 & 1 \\
0 & 0 & 1 & 1 & 1 \\
0 & 0 & 0 & 1 & 1
\end{array}\right), M\left(H^{E P}\right)=\left(\begin{array}{ccc}
0 & 0 & 0 \\
0 & 1 & 0 \\
0 & 0 & 1 \\
1 & 0 & 1 \\
1 & 1 & 1
\end{array}\right) .
$$

The usefulness of the edge power complement to us lies in the following corollary.

Corollary 4.4. Suppose $q \geq 0$ and $0 \leq n \leq 2^{q}$. There exists an asymmetric n-uniform hypergraph with $q+1$ edges if and only if there exists an asymmetric $\left(2^{q}-n\right)$-uniform hypergraph with $q+1$ edges.

Proof. From Corollary 4.2 we have that $H$ is an asymmetric $n$-uniform hypergraph with $q+1$ edges if and only if $H^{E P}$ is an asymmetric $\left(2^{q}-n\right)$-uniform hypergraph with $q+1$ edges. 
We are now able to improve the lower bound of Corollary 3.2.

Corollary 4.5. If $m \geq 1, n \geq 3$, and $H$ is an asymmetric $n$-uniform hypergraph with $m \geq 1$ edges, then $m \geq\left\lfloor\log _{2}(n+1)\right\rfloor+2$. Moreover, if $n=6$ then $m \geq 5$, and if $n=14$ then $m \geq 6$.

Proof. We have $2^{q-1}+1 \leq n \leq 2^{q}$, with $q \geq 2$. The bound from Corollary 3.2 is $m \geq q+1$, which is identical with the bound here except when $n=2^{q}$ or $2^{q}-1$, or $2^{q}-2$ with $q=3$ or 4 (i.e., $n=6$ or 14). In those cases the bound here is $m \geq q+2$, so we must show that $H$ cannot have $q+1$ edges. If $H$ did have $q+1$ edges then we could apply Corollary 4.4 to obtain a 0 -, 1 - or 2 -uniform asymmetric hypergraph with $q+1$ edges, contradicting Theorem 3.3.

\section{Constructions for $n=2^{q}, 2^{q}-1,2^{q}-2$}

Corollary 4.4 presents part of an inductive construction for asymmetric uniform hypergraphs. However, Theorem 3.3 leaves some holes in this inductive process, so we will need constructions for $n=2^{q}$ and $n=2^{q}-1$ for all $q \geq 2$. Additionally, we need special constructions for $n=3,6$, and 14 .

Lemma 5.1. If $n=2^{q}$ for $q \geq 2$, then there exists an asymmetric $n$-uniform hypergraph with $q+2$ edges.

Proof. Let $V(H)$ be all possible binary $(q+1)$-tuples $x=\left(x_{0}, x_{1}, x_{2}, \ldots, x_{q}\right)$, and set $S_{i}=\left\{x \in V(H) \mid x_{i}=1\right\}, 0 \leq i \leq q$. Let $T=\overline{S_{0}}=\left\{x \in V(H) \mid x_{0}=0\right\}$. We have $\left|S_{i}\right|=|T|=2^{q}$ for all $i$. Furthermore, $\left|S_{i} \cap S_{j}\right|=2^{q-1}$ for all $i \neq j,\left|S_{i} \cap T\right|=2^{q-1}$ for all $i \geq 1$, and $\left|S_{0} \cap T\right|=0$. For $1 \leq k \leq q-1$, let $v(k) \in T$ be the vertex with $v(k)_{k}=1$ and $v(k)_{\ell}=0$ for all $\ell \neq k$; also, let $w(k) \notin T$ be the vertex with $w(k)_{0}=1, w(k)_{\ell}=0$ for $1 \leq \ell \leq k-1$, and $w(k)_{\ell}=1$ for $k \leq \ell \leq q$. Set $T^{\prime}=T \backslash\{v(1), v(2), \ldots, v(q-1)\} \cup$ $\{w(1), w(2), \ldots, w(q-1)\}$. Set $E(H)=\left\{S_{0}, S_{1}, \ldots, S_{q}, T^{\prime}\right\}$. We claim the set structure $H$ is an asymmetric $2^{q}$-uniform hypergraph with $q+2$ edges. The uniformity and size of $E(H)$ are clear from construction. To show there are no automorphisms, we consider each edge's intersection with $T^{\prime}$. To get from $T$ to $T^{\prime}$ we replaced $q-1$ vertices with $v(k)_{0}=0$ with $q-1$ vertices with $w(k)_{0}=1$, so that $\left|S_{0} \cap T^{\prime}\right|=q-1$. For $i \geq 1, v(k)_{i}=0$ and $w(k)_{i}=1$ for $1 \leq k \leq i-1$, while $v(k)_{i}=w(k)_{i}$ for $k \geq i$, so that $\left|S_{i} \cap T^{\prime}\right|=2^{q-1}+i-1$. Since $2^{q-1}+i-1>q-1$ for all $i \geq 1$ and $q \geq 2$, the size of the intersection $S_{i} \cap T^{\prime}$ is unique for every edge $S_{i}$. $T^{\prime}$ is the only edge to have all unique intersection sizes, so any automorphism of $H$ must fix $T^{\prime}$. But this also fixes every $S_{i}$ according to the size of its intersection with $T^{\prime}$. Thus, there are no nontrivial edge automorphisms of $H$. Since $H$ is clearly vertex-simple, by Lemma 2.2(2) $H$ is asymmetric.

Corollary 5.2. If $n=2^{q}-1$ for some integer $q \geq 2$, then there exists an asymmetric $n$-uniform hypergraph with $q+2$ edges.

Proof. In the construction of $H$ above, $w(1)=(1,1, \ldots, 1)$ is incident with every edge. Thus, removing it creates an edge-simple $\left(2^{q}-1\right)$-uniform hypergraph $H-w(1)$ with $q+2$ edges. Any (vertex) automorphism $\pi$ of $H-w(1)$ can be extended to an automorphism of $H$ by setting $\pi(w(1))=w(1)$, so $H-w(1)$ must be asymmetric as well.

The construction in Corollary 5.2 is valid when $n=3$, but gives a hypergraph with 3 vertices of degree 1. Later we will need a hypergraph with fewer vertices of degree 1. 
Lemma 5.3. There exist asymmetric hypergraphs $H$ as follows.

1. $H$ is 3-uniform with 4 edges, and 2 vertices of degree 1.

2. $H$ is 6-uniform with 5 edges.

3. $H$ is 14-uniform with 6 edges.

Proof. For each proof below, the transpose of the incidence matrix, $M(H)^{T}$, is given. The columns (vertices) are indexed by $v_{1}, v_{2}, \ldots, v_{2 n}$, where $n=3,6$ and 14 , respectively, and the rows (edges) are indexed by $e_{1}, e_{2}, \ldots, e_{m}$, where $m=4,5$, and 6 , respectively. To prove the induced hypergraph $H$ is asymmetric, the sizes of the edge intersections are provided in a second matrix $A=\left(a_{i j}\right)$, with $a_{i j}=\left|e_{i} \cap e_{j}\right|$ (alternatively, $A=M(H)^{T} M(H)$ ). If two rows (edges) $e_{i}$ and $e_{j}$ have a different multiset of intersection sizes, then there can be no (edge) automorphism $\sigma$ such that $\sigma\left(e_{i}\right)=e_{j}$. If all rows of $A$ consist of distinct multisets, then the automorphism group is trivial. This covers parts (ii) and (iii) below, while an additional argument is needed for part (i).

(1)

$$
M(H)^{T}=\left(\begin{array}{llllll}
1 & 1 & 1 & 0 & 0 & 0 \\
1 & 1 & 0 & 1 & 0 & 0 \\
1 & 0 & 0 & 1 & 1 & 0 \\
0 & 0 & 1 & 1 & 0 & 1
\end{array}\right), A=\left(\begin{array}{llll}
3 & 2 & 1 & 1 \\
2 & 3 & 2 & 1 \\
1 & 2 & 3 & 1 \\
1 & 1 & 1 & 3
\end{array}\right)
$$

From $A$ it might still be possible for an edge automorphism to swap $e_{1}$ and $e_{3}$. However, $e_{3}$ contains a vertex of degree 1 , while $e_{1}$ does not; thus, no such automorphism exists. Note that $v_{5}$ and $v_{6}$ are the vertices of degree 1 .

(2)

$$
\begin{aligned}
M(H)^{T}= & \left(\begin{array}{cccccccccccc}
1 & 1 & 1 & 1 & 1 & 1 & 0 & 0 & 0 & 0 & 0 & 0 \\
1 & 1 & 1 & 0 & 0 & 0 & 1 & 1 & 1 & 0 & 0 & 0 \\
1 & 1 & 0 & 1 & 0 & 0 & 1 & 1 & 0 & 1 & 0 & 0 \\
1 & 0 & 0 & 1 & 1 & 0 & 1 & 0 & 0 & 1 & 1 & 0 \\
1 & 0 & 0 & 0 & 0 & 0 & 1 & 1 & 0 & 1 & 1 & 1
\end{array}\right), \\
A & =\left(\begin{array}{ccccc}
6 & 3 & 3 & 3 & 1 \\
3 & 6 & 4 & 2 & 3 \\
3 & 4 & 6 & 4 & 4 \\
3 & 2 & 4 & 6 & 4 \\
1 & 3 & 4 & 4 & 6
\end{array}\right) .
\end{aligned}
$$

(3)

$$
\begin{aligned}
M(H)^{T}=\left(\begin{array}{cccccccccccccc}
1 & 1 & 1 & 1 & 1 & 1 & 1 & 1 & 1 & 1 & 1 & 1 & 1 & 1 \\
1 & 1 & 1 & 1 & 1 & 1 & 1 & 0 & 0 & 0 & 0 & 0 & 0 & 0 \\
1 & 1 & 1 & 1 & 0 & 0 & 0 & 1 & 1 & 1 & 1 & 0 & 0 & 0 \\
1 & 1 & 0 & 0 & 1 & 0 & 0 & 1 & 1 & 0 & 0 & 1 & 1 & 0 \\
1 & 0 & 1 & 0 & 0 & 1 & 0 & 1 & 0 & 1 & 0 & 1 & 0 & 0 \\
1 & 0 & 0 & 0 & 0 & 0 & 0 & 0 & 0 & 0 & 0 & 0 & 0 & 0 \\
1 & 1 & 1 & 1 & 1 & 1 & 1 & 0 & 0 & 0 & 0 & 0 & 0 & 0 \\
1 & 1 & 1 & 0 & 0 & 0 & 0 & 1 & 1 & 1 & 0 & 0 & 0 & 0 \\
1 & 0 & 0 & 1 & 1 & 0 & 0 & 1 & 1 & 0 & 1 & 1 & 0 & 0 \\
1 & 1 & 0 & 1 & 0 & 1 & 0 & 1 & 0 & 1 & 1 & 0 & 1 & 0 \\
1 & 1 & 1 & 1 & 1 & 1 & 0 & 1 & 1 & 1 & 1 & 1 & 1 & 1
\end{array}\right),
\end{aligned}
$$




$$
A=\left(\begin{array}{cccccc}
14 & 7 & 8 & 7 & 6 & 1 \\
7 & 14 & 7 & 6 & 7 & 7 \\
8 & 7 & 14 & 7 & 8 & 7 \\
7 & 6 & 7 & 14 & 7 & 8 \\
6 & 7 & 8 & 7 & 14 & 9 \\
1 & 7 & 7 & 8 & 9 & 14
\end{array}\right)
$$

\section{Main result}

Our overall argument proceeds by induction on $n$, but for each $n$ we also need to apply induction on $m$, as provided by the following lemma.

Lemma 6.1. Suppose that $m \geq 1$ and $n \geq 2$. If there exists an asymmetric $n$-uniform hypergraph with $m$ edges and at most $n-1$ vertices of degree 1 , then there exists an asymmetric n-uniform hypergraph with $m^{\prime}$ edges for all $m^{\prime} \geq m$.

Proof. Let $H$ be an asymmetric $n$-uniform hypergraph with $m$ edges and vertices $v_{1}, v_{2}$, $\ldots, v_{k}$ of degree 1 , where $k \leq n-1$. We may assume $H$ has no vertex of degree 0 (any such vertex may be deleted). Form the edge $e=\left\{w, v_{1}, v_{2}, \ldots, v_{k}, x_{1}, \ldots, x_{n-k-1}\right\}$, where $w \notin V(H)$, and the $x_{i}$ 's are any other distinct vertices in $V(H)$. The hypergraph $H+e$ contains only one vertex of degree 1 , namely $w$. The edge $e$ containing $w$ must be fixed under any automorphism, and it follows that any edge automorphism of $H+e$ must also be an edge automorphism of $H$. Thus, the only automorphism of $H+e$ is the trivial one, and we have an asymmetric $n$-uniform hypergraph with $m+1$ edges. Since we now have only one vertex of degree 1 , we can repeat this process to get an asymmetric $n$-uniform hypergraph with $m^{\prime}$ edges for all $m^{\prime} \geq m$.

Remark 6.2. Since a vertex-simple hypergraph with $m$ edges can have at most $m$ vertices of degree 1 , the degree 1 requirement holds automatically for $m \leq n-1$.

Theorem 6.3. Let $m, n$ be integers with $m \geq 1$ and $n \geq 2$. There exists an asymmetric $n$-uniform hypergraph with $m$ edges if and only if $m \geq f(n)$, where:

$$
f(n)= \begin{cases}6 & \text { if } n=2 \\ 5 & \text { if } n=6 \\ 6 & \text { if } n=14 \\ \left\lfloor\log _{2}(n+1)\right\rfloor+2 & \text { if } n>2, n \neq 6,14\end{cases}
$$

Proof. The case $n=2$ is covered by Theorem 3.3(2). For $n \geq 3$, applying Corollary 4.5 and Lemma 6.1 means it suffices to find such a hypergraph for $m=f(n)$ with at most $n-1$ vertices of degree 1 . Since $f(n) \leq n-1$ for all $n \geq 5$, Remark 6.2 says we can ignore the degree 1 requirement except when $n=3$ or 4 . We proceed by induction on $n$. Note that for $3 \leq k \leq n$ we always have $f(k) \leq f(n)$.

Case 1. If $2^{q-1}+1 \leq n \leq 2^{q}-2$ with $q \geq 3$ and $n \neq 6$ or 14 , then let $n=2^{q}-k$ with $2 \leq k \leq 2^{q-1}-1<n$. Since $f(k) \leq f(n)$ (when $k=2$ this follows because $n \neq 6$ or 14), by the inductive assumption there exists an asymmetric $k$-uniform hypergraph with $f(n)=q+1$ edges. Applying Corollary 4.4 provides the desired $n$-uniform hypergraph.

Case 2. The cases $n=6$ and 14 follow from Lemma 5.3(2) and (3). 
Case 3. If $n=2^{q}-1$ with $q \geq 3$, then the result follows from Corollary 5.2.

Case 4. If $n=3$, then the result follows from Lemma 5.3(1).

Case 5. If $n=2^{q}$ with $q \geq 2$, then the result follows from Lemma 5.1. Note that for $n=4$ the construction produces only 3 vertices of degree 1 .

This exhausts all cases and completes the proof.

Corollary 6.4. Let $m, n$ be integers with $m \geq 1$ and $n \geq 2$, and let $f(n)$ be defined as in Theorem 6.3.

1. There exists a distinguishing partition of $K_{m(n)}$ if and only if $m \geq f(n)$.

2. There exists a distinguishing partition of $m K_{n}$ if and only if $m \geq f(n)$.

3. There exists an asymmetric $n$-regular hypergraph with $m$ vertices if and only if $m \geq$ $f(n)$.

4. There exists a distinguishing partition for the wreath product $S_{n}$ Wr $S_{m}$ acting on $[n] \times[m]$ if and only if $m \geq f(n)$.

Proof. Apply Theorem 3.1 to Theorem 6.3.

\section{Final comments}

An obvious next step would be to try to extend our main result to other complete multipartite graphs. Dealing with all complete multipartite graphs may be difficult, so it may be sensible to focus on some special classes.

If we fix $m$, there are only finitely many complete $m$-partite graphs with a distinguishing partition. Arguments similar to those elsewhere in this paper show that a distinguishing partition has at most $2^{m}-1$ parts, and each part has size at most $m$, so if the total number of vertices is large there cannot be a distinguishing partition. (Better bounds on the number of vertices could be obtained, but we just wish to establish the general idea.) For example, for complete bipartite graphs it is not hard to show the following.

Proposition 7.1. The only complete bipartite graph $K_{r, s}, 1 \leq r \leq s$, with a distinguishing partition is $K_{1,2}$.

It therefore seems more interesting to look at complete multipartite graphs $K_{n_{1}, n_{2}, \ldots, n_{m}}$ with arbitrarily large $m$, but where $n_{1}, n_{2}, \ldots, n_{m}$ take only a small number of distinct values.

\section{Acknowledgements}

The first author would like to thank Tom Tucker for helpful conversations, and we thank Melody Chan for providing references [11, 14].

\section{References}

[1] M. O. Albertson and K. L. Collins, Symmetry breaking in graphs, Electron. J. Combin. 3 (1996), \#R18.

[2] B. Bogstad and L. J. Cowen, The distinguishing number of the hypercube, Discrete Math. 283 (2004), 29-35. 
[3] P. J. Cameron, Permutation Groups, in Handbook of Combinatorics, Vol. 1, Elsevier, Amsterdam, 1995, 611-645.

[4] M. Chan, The distinguishing number of the augmented cube and hypercube powers, Discrete Math. 308 (2008), 2330-2336.

[5] M. Chan, The distinguishing number of the direct product and wreath product action, J. Algebraic Combin. 24 (2006), 331-345.

[6] M. Chan, The maximum distinguishing number of a group, Electron. J. Combin. 13 (2006), \#R70.

[7] M. J. Fisher and G. Isaak, Distinguishing colorings of Cartesian products of complete graphs, Discrete Math. 308 (2008), 2240-2246.

[8] A. Gardiner, Homogeneous graphs, J. Combinatorial Theory Ser. B 20 (1976) 94-102.

[9] W. Imrich, J. Jerebic, and S. Klavžar, The distinguishing number of Cartesian products of complete graphs, European J. Combin. 29 (2008), 922-929.

[10] W. Imrich and S. Klavžar, Distinguishing Cartesian powers of graphs, J. Graph Theory 53 (2006), 250-260.

[11] S. Klavžar, T.-L. Wong, and X. Zhu, Distinguishing labellings of group action on vector spaces and graphs, J. Algebra 303 (2006), 626-641.

[12] S. Klavžar and X. Zhu, Cartesian powers of graphs can be distinguished by two labels, European J. Combin. 28 (2007), 303-310.

[13] J. Tymoczko, Distinguishing numbers for graphs and groups, Electron. J. Combin. 11 (2004), \#R63.

[14] T.-L. Wong and X. Zhu, Distinguishing labeling of group actions, Discrete Math. 309 (2009), 1760-1765. 